\title{
Prognostic value of new left atrial volume index severity partition cutoffs after cardiac rehabilitation program in patients undergoing cardiac surgery
}

Davide Lazzeroni ${ }^{i^{*}}$, Nicola Gaibazzi ${ }^{2}$, Matteo Bini ${ }^{3}$, Giacomo Bussolati ${ }^{1}$, Umberto Camaiora ${ }^{1}$, Roberto Cassi ${ }^{1}$, Simone Geroldi ${ }^{1}$, Pietro Tito Ugolotti ${ }^{1}$, Lorenzo Brambilla ${ }^{1}$, Valerio Brambilla ${ }^{1}$, Paolo Castiglioni ${ }^{4}$ and Paolo Coruzzi ${ }^{3}$

\begin{abstract}
Background: Previous studies showed that left atrial enlargement is an independent marker of adverse outcomes in both primary and secondary cardiovascular prevention. However, no data are available on long-term outcomes in patients undergoing valve surgery and/or coronary artery by-pass graft (CABG) surgery. Aim of the study was to evaluate long-term prognostic role of left atrial volume index (LAVi) after cardiac surgery, using the cutoff values recently proposed by the European Association of Cardiovascular Imaging and American Society of Echocardiography.

Methods: We created a retrospective registry of 1703 consecutive patients who underwent cardiovascular rehabilitation program after cardiac surgery, including CABG, valve surgery and valve + CABG surgery. LAVi was calculated as ratio of left atrium volume to body surface area, in $\mathrm{ml} / \mathrm{m}^{2}$ at discharge; 563 patients with available LAVi data were included in the study.

Results: In the whole population LAVi was $36 \pm 14 \mathrm{ml} / \mathrm{m}^{2}$ (mean \pm SD) and the follow-up time was $5 \pm 1$. 5 years. Increased LAVi $\left(>34 \mathrm{ml} / \mathrm{m}^{2}\right)$ predicted major adverse cardiovascular and cerebrovascular events (MACCEs) (HR= 2.1; Cl95 \%: 1.4-3.1; $p<0.001)$ and cardiovascular mortality (HR=2.2; Cl95 \%: 1.0-4.5; $p=0.032)$. An increased LAVi remained MACCEs predictor after adjustement for age, gender, diabetes, atrial fibrillation at discharge, echocardiographic E/A ratio and left ventricular ejection fraction (HR=1.8; Cl95\%: 1.0-3.0; $p=0$. 036). When the study population was split according to increasing LAVi values, left atrium enlargement resulted a predictor of progressively worse adverse outcome.
\end{abstract}

Conclusions: LAVi is a predictor of long-term adverse cardiovascular outcome after cardiac surgery, even after correction for main clinical and echocardiographic variables. The recently recommended LAVi severity cutoffs appear adequate to effectively stratify outcome in patients undergoing rehabilitation after cardiac surgery.

Keywords: Left atrial volume index, Coronary artery by-pass graft, Cardiac valve surgery, Echocardiography, cardiovascular outcomes

Abbreviations: ACS, Acute coronary syndrome; CABG, Coronary artery by-pass graft; CRP, Cardiac rehabilitation program; E/A, Ratio of the early to late ventricular filling velocities; EF, Ejection fraction; $H F$, Heart failure; LA, Left atrium; LAVi, Left atrial volume index; LV, Left ventricle; MACCEs, Major adverse cardiovascular and cerebrovascular events; MI, Myocardial infarction

\footnotetext{
* Correspondence: davide.lazzeroni@gmail.com

${ }^{1}$ Fondazione Don Carlo Gnocchi, University of Parma, Fondazione Don

Gnocchi, Piazzale dei servi n 3, 43121 Parma, Italy

Full list of author information is available at the end of the article
} 


\section{Background}

Left atrium (LA) modulates left ventricular (LV) filling and cardiovascular performance by functioning as a reservoir and a conduit for pulmonary venous return and as a "booster" that augments ventricular filling during late ventricular diastole. LA also reflects LV filling pressure and is capable of remodeling (enlarging) in response to its elevation $[1,2]$. Although there is a growing body of evidence demonstrating that LA enlargement is an independent marker of adverse outcomes both in primary [3-7] and secondary [8-11] cardiovascular prevention, to date, there are no published data assessing long-term outcome in patients undergoing valve and coronary artery by-pass graft (CABG) surgery.

Aim of this retrospective study was to examine longterm prognostic implications of left atrial volume index (LAVi) assessment in patients undergoing cardiovascular rehabilitation program (CRP) after cardiac surgery, using the cutoff values proposed by the recently-published guidelines of the European Association of Cardiovascular Imaging (EACVI) and American Society of Echocardiography (ASE) [12].

\section{Methods}

\section{Patients' selection}

We created a registry of consecutive patients undergoing cardiovascular rehabilitation program (CRP) after cardiac surgery, including $C A B G$, valve surgery and combined valve and CABG surgery, from January 2007 to June 2012. A total of 1703 subjects (70\% men, mean age of $69 \pm$ 11 years) were collected and, out of them, all 563 patients with available biplane left atrial volume data were extracted for the current analysis. The internal review board of Fondazione Don Gnocchi approved data collection. All patients completed a standard in-hospital CRP, lasting approximately 2 weeks, consisting of supervised exercise sessions (120 min per day), lifestyle and risk factor management, medical counseling and medical therapy optimization. For each patient we collected variables from medical record including anamnestic and demographic findings, clinical and laboratory markers, electrocardiographic and echocardiographic measurements, physical activity data, pharmacological therapy adherence. Outcomes were collected through a telephonic questionnaire administered by a medical doctor or a supervised fellow and all patients provided oral informed consent. End points were: overall mortality and major adverse cardiovascular and cerebrovascular events (MACCEs) defined as either: cardiovascular (CV) mortality, non-fatal acute coronary syndrome (ACS), heart failure (HF) and stroke.

\section{Echocardiographic data}

M-mode and 2-dimensional echocardiographic images were obtained with a commercially available echocardiography machine (Esaote $\mathrm{MyLab}^{\mathrm{Tm}}$ 60). LA volume was calculated using the biplane area-length method at discharge of CRP [12]. Measurements were obtained in end-systole from the frame preceding mitral valve opening. LAVi was calculated as the ratio of LA volume to body surface area $\left(\mathrm{ml} / \mathrm{m}^{2}\right)$. In agreement with ASE and EACVI guidelines, we assumed $\mathrm{LAVi}>34 \mathrm{ml} / \mathrm{m}^{2}$ as the LA enlargement threshold and we further classified LAVi values from 35 to $41 \mathrm{ml} / \mathrm{m}^{2}$ as mild LA enlargement, from 42 to $48 \mathrm{ml} / \mathrm{m}^{2}$ as moderate LA enlargement and greater than $48 \mathrm{ml} / \mathrm{m}^{2}$ as severe enlargement [12].

\section{Statistical analysis}

Continuous variables were expressed as mean (M) and standard deviation (SD), categorical variables as number $(\mathrm{N})$ and percentage (\%). The Student 2-sample $t$ test, Pearson $\chi 2$ test, Mann-Whitney-Wilcoxon rank-sum test were used to compare the differences between groups for continuous, categorical and ordinal variables, respectively. Cox proportional hazard regression analysis was performed to create survival-free-from-event curves. Multivariate Cox regression analysis was used to create adjustment of hazard ratio (HR) for age, left ventricular ejection fraction (LVEF), gender, diabetes, and ratio of the early (E) to late (A) ventricular filling velocities (E/A ratio). Event-free survival analysis was measured from admission to the first event. Significance was defined as a $p$ value $<0.05$. All statistics were performed with SPSS version 21 (IBM Corporation).

\section{Results}

\section{Demographic and clinical characteristics}

A total of 563 patients were included in the study. Mean age was $69 \pm 10$ years and male gender was prevalent, with 399 males (70 \%). 149 patients (26\%) were affected by type 2 diabetes and 305 patients $(54 \%)$ by arterial hypertension. 292 patients (52\%) underwent CABG, 191 (34 \%) valve surgery, 80 (14\%) combined valve and CABG surgery.

One hundred and eighty-four patients (33\%) underwent cardiac revascularization after ACS and $98(18 \%)$ after stable angina or silent ischemia; 100 patients (18\%) were affected by mitral valve regurgitation, 9 (2\%) by mitral valve stenosis, 73 (13\%) by aortic valve stenosis and $41(7 \%)$ by aortic valve regurgitation. LAVi and LVEF mean values over the whole group of patients were $36 \pm 14 \mathrm{ml} / \mathrm{m}^{2}$ and $51 \pm 9 \%$, respectively.

LA enlargement was found in 257 patients (46\%), 97 (17 \%) patients with mild LA enlargement, 71 (13\%) with moderate LA enlargement and 89 (16\%) with severe LA enlargement.

Patients with LA enlargement were significantly older compared with patients with normal LA $(71 \pm 9$ vs $68 \pm$ 
10 years, $<p=0.001$ ). A higher prevalence of normal LA was observed in the subgroup of patients with established coronary artery disease such as prior acute coronary syndrome (normal LA $63 \%$ vs LA enlargement $37 \%$; $p=0.001$ ) or stable angina (normal LA $67 \%$ vs LA enlargement $33 \% ; p=0.002)$. Conversely, LA enlargement was more frequent in the subgroup of patients with mitral regurgitation (normal LA $44 \%$ vs LA enlargement $56 \% ; p=0.034$ ) and aortic stenosis (normal LA $32 \%$ vs LA enlargement $68 \% ; p<0.001$ ). Mean LVEF was significantly lower in patients with LAVi > $34 \mathrm{ml} / \mathrm{m}^{2}$ in comparison to the group with normal LAVi $(49 \pm 10 \%$ vs $52 \pm 9 \% ; p<0.005)$. Sinus rhythm rate at discharge was higher in patients with normal LAVi ( $59 \%$ vs $41 \% ; p<0.01$ ) and no differences in the use of beta-blockers (52\% vs $48 \% ; p=$ n.s.) and amiodarone (51 \% vs $49 \%$; $p=$ n.s.) were found between normal LAVi and LAVi enlargement; conversely, statins and ace-inhibitors administration was higher in patients with normal LAVi (56\% vs $44 \%$; $p<0.01$ and $57 \%$ vs $43 \%$; $p<0.01$ respectively).

Table 1 shows differences in baseline parameters between normal LA and LA enlargement.

\section{End point}

Mean follow-up was $5 \pm 1.5$ years. All-cause mortality consisted in 55 deaths $(10 \%)$ and the total amount of MACCEs was 114 (20\%), composed by $31 \mathrm{CV}$ deaths (5\%), 43 HF hospitalization (8\%), 15 strokes (3\%) and $25 \operatorname{ACS}(4 \%)$.

MACCEs were 2.1-fold higher in patients with LAVi > $34 \mathrm{ml} / \mathrm{m}^{2}$ compared with patients with normal LA volume, this difference being significant even considering patients who received CABG or valve surgery separately (in particular in patients with mitral and aortic regurgitation; see Table 2). Moreover, event-free survival from MACCEs was significantly lower in both patients who underwent CABG and valve surgery with enlarged LA (Fig. 1).

By dividing the whole population according to severity partition cut-offs, MACCEs rate was significantly higher in patients with either moderate or severe LA enlargement but not in those with mild LA enlargement (Fig. 2 and Table 2). Multivariate analysis, adjusted for age, gender, diabetes, E/A, atrial fibrillation at discharge and LVEF, confirmed the role of LA enlargement in predicting MACCEs in the whole population; moreover, the progressive increase of LAVi severity maintained an incremental risk of MACCEs in patients with LA enlargement ranging from moderate to severe (Fig. 3).

Severe LA enlargement resulted a significant and robust predictor of any type of secondary end point: all-
Table 1 Baseline characteristics

\begin{tabular}{|c|c|c|}
\hline \multirow[t]{2}{*}{ Parameters } & \multirow{2}{*}{$\begin{array}{l}\text { Normal LA } \\
(\mathrm{LAVi} \leq 34 \mathrm{ml} / \mathrm{m} 2) \\
N=306\end{array}$} & \multirow{2}{*}{$\begin{array}{l}\text { LA Enlargement } \\
(\mathrm{LAVi}>34 \mathrm{ml} / \mathrm{m} 2) \\
N=257\end{array}$} \\
\hline & & \\
\hline Age (years) & $68(10)$ & $71(9)^{* *}$ \\
\hline Male sex & $245(61)$ & $154(39)^{* *}$ \\
\hline $\mathrm{BMI}\left(\mathrm{kg} / \mathrm{m}^{2}\right)$ & $26.9(4)$ & $26.7(4)$ \\
\hline $\mathrm{BSA}\left(\mathrm{m}^{2}\right)$ & $1.9(0.2)$ & $1.8(0.2)^{* *}$ \\
\hline \multicolumn{3}{|l|}{ CV risk Factors } \\
\hline Familiar history (CVD) & $159(56)$ & $120(44)$ \\
\hline AHT & $159(52)$ & $146(48)$ \\
\hline DM & $83(55)$ & $66(45)$ \\
\hline Dyslipidaemia & $165(57)$ & $120(43)$ \\
\hline \multicolumn{3}{|l|}{ Types of cardiac surgery } \\
\hline CABG & $197(75)$ & $95(25)^{* *}$ \\
\hline Heart-valve surgery & $73(25)$ & $118(75)^{* *}$ \\
\hline CABG + Valve surgery & $37(46)$ & $43(54)$ \\
\hline \multicolumn{3}{|l|}{ Clinical indications } \\
\hline ACS & $116(63)$ & $68(37)^{*}$ \\
\hline Stable Angina & $66(67)$ & $32(33)^{*}$ \\
\hline MV Stenosis & $2(22)$ & $7(78)$ \\
\hline MV regurgitation & $44(44)$ & $56(56)^{*}$ \\
\hline AV Stenosis & $23(32)$ & $20(68)^{* *}$ \\
\hline $\mathrm{AV}$ regurgitation & $19(46)$ & $22(54)$ \\
\hline Other indication & $36(62)$ & $22(38)$ \\
\hline \multicolumn{3}{|c|}{ Echocardiographic parameters } \\
\hline LAV (ml) & $49(11)$ & $85(22)^{* *}$ \\
\hline $\mathrm{LAd}(\mathrm{mm})$ & $38(5)$ & $54(6)^{* *}$ \\
\hline LVEF (\%) & $52(9)$ & $49(10)^{*}$ \\
\hline LV EDV (ml) & $105(41)$ & $108(42)$ \\
\hline IVS (mm) & $12(2)$ & $13(2)^{* *}$ \\
\hline E/A Ratio & $1(0.4)$ & $1(0.6)$ \\
\hline \multicolumn{3}{|l|}{ Medications } \\
\hline$\beta$-Blocker & $201(52)$ & $185(48)$ \\
\hline Amiodarone & $79(51)$ & 77 (49) \\
\hline OAT & $72(38)$ & $117(62)^{* *}$ \\
\hline ACEi & $216(57)$ & $170(43)^{* *}$ \\
\hline Statins & $218(56)$ & $168(44)^{* *}$ \\
\hline
\end{tabular}

Values are expressed as mean and standard deviation or as number and percentage

$L A$ left atrium; $L A V i$ left atrial volume index; $B M I$ body mass index; $C V D$ cardiovascular disease; $A H T$ arterial hypertension; $D M$ diabetes mellitus; $C A B G$ coronary artery bypass graft; $A C S$ acute coronary syndrome; $M V$ mitral valve; $A V$ aortic valve; $L a d$ left atrium antero-posteior diameter; $L V E F$ left ventricular ejection fraction; IVSs interventricular septum; LV EDV left ventricular enddiastolic volume; $O A T$ oral anticoagulant therapy

*and **indicate differences at significance levels $p<0.05$ and $p<0.01$ respectively

cause mortality, $\mathrm{CV}$ mortality, $\mathrm{HF}$ and stroke. In patients with moderate LA enlargement, HF rate was 2.5 -fold higher in comparison with normal LA size group. 
Table 2 HR for primary and secondary end points by univariate Cox proportional hazard regression analysis

\begin{tabular}{|c|c|c|c|c|c|c|c|c|}
\hline \multirow[b]{3}{*}{ MACCES } & & & \multicolumn{6}{|c|}{ LA severity partition cut-off } \\
\hline & \multicolumn{2}{|c|}{ LA enlargement (LAVi > 34 ml/m2) } & \multicolumn{2}{|c|}{ Mild LA enlargement } & \multicolumn{2}{|c|}{ Moderate LA enlargement } & \multicolumn{2}{|c|}{ Severe LA enlargement } \\
\hline & $\mathrm{HR}(95 \% \mathrm{Cl})$ & $\mathrm{p}$ & HR $(95 \% \mathrm{Cl})$ & $p$ & $\mathrm{HR}(95 \% \mathrm{Cl})$ & $p$ & $\mathrm{HR}(95 \% \mathrm{Cl})$ & $p$ \\
\hline All Patients & $2.1(1.4-3.1)$ & $0.000 \#$ & $1.2(0.7-2.2)$ & 0.457 & $2.2(1.3-2.7)$ & $0.003^{*}$ & $3.1(2.0-4.9)$ & $0.000 \#$ \\
\hline CABG Patients & $2.1(1.3-3.2)$ & $0.001^{*}$ & $1.8(0.7-4.5)$ & 0.201 & $3.2(1.2-7.9)$ & $0.013^{*}$ & $6.1(2.6-14.1)$ & 0.000\# \\
\hline Valve Patients & $2.7(1.2-6.0)$ & $0.011^{*}$ & $1.6(0.5-4.9)$ & 0.402 & $2.9(1.1-7.7)$ & $0.025^{*}$ & $3.3(1.4-8.0)$ & $0.005 \#$ \\
\hline Aortic stenosis & $6.6(0.8-51)$ & 0.071 & $0.1(0.1-1.0)$ & 0.521 & $1.9(0.3-11)$ & 0.482 & $1.6(0.2-9.6)$ & 0.060 \\
\hline Aortic regurgitation & $4.2(1.5-11)$ & $0.004^{*}$ & $3.3(0.7-15)$ & 0.117 & $3.7(1.1-12)$ & $0.028^{*}$ & $4.5(1.5-13)$ & $0.006^{*}$ \\
\hline Mitral regurgitaton & $4.2(1.5-11)$ & $0.004^{*}$ & $1.9(0.1-31)$ & 0.638 & $6.4(0.7-57)$ & 0.096 & $7.0(0.9-57)$ & 0.067 \\
\hline Valve + CABG & $2.3(0.9-5.6)$ & 0.053 & $1.6(0.4-6.3)$ & 0.484 & $2.4(0.7-7.8)$ & 0.122 & $2.6(0.9-7.1)$ & 0.050 \\
\hline All-cause mortality & $1.6(0.9-2.8)$ & 0.064 & $1.2(0.5-2.7)$ & 0.533 & $1.6(0.7-3.5)$ & 0.258 & $2.2(1.1-4.2)$ & $0.025^{*}$ \\
\hline CV mortality & $2.2(1.0-4.5)$ & $0.032^{*}$ & $1.2(0.4-3.7)$ & 0.739 & $2.5(0.9-6.6)$ & 0.070 & $3.1(1.3-7.4)$ & $0.010^{*}$ \\
\hline Heart failure & $1.8(0.9-3.5)$ & 0.057 & $0.8(0.2-2.5)$ & 0.796 & $2.5(1.0-5.8)$ & $0.034^{*}$ & $2.5(1.1-5.4)$ & $0.023^{*}$ \\
\hline \multirow[t]{2}{*}{ Stroke } & $1.6(0.5-4.8)$ & 0.327 & $1.0(0.2-5.3)$ & 0.918 & n.a. & n.a. & $3.8(1.2-11.9)$ & $0.019^{*}$ \\
\hline & & & & & n.a. & & & \\
\hline ACS & $1.9(0.8-4.4)$ & 0.137 & $1.5(0.4-4.9)$ & 0.492 & $1.6(0.4-5.9)$ & 0.483 & $2.6(0.9-7.4)$ & 0.067 \\
\hline
\end{tabular}

MACCEs major adverse cardiovascular and cerebrovascular events; $L A$ left atrial; LAVi left atrial volume index; $H R$ hazard ratio; CI confidence interval; CABG coronary artery by-pass graft; Valve valve surgery; $C V$ cardiovascular; $A C S$ acute coronary syndrome; n.a not applicable (no event)

$P$ value: difference in comparison with normal LAVi group $(\leq 34 \mathrm{ml} / \mathrm{m} 2) * p<0.05 ; \# p<0.001$

Outcomes of patients with normal LAVi and with mild LA enlargement did not differ (Table 2).

Cardiovascular mortality rate was 2.2 -fold higher in patients with $\mathrm{LAVi}>34 \mathrm{ml} / \mathrm{m}^{2}$. Event-free survival from cardiovascular mortality was significantly lower in these patients (Fig. 4), whilst only a trend towards a higher all-cause mortality was found in patients with $\mathrm{LAVi}>34 \mathrm{ml} / \mathrm{m}^{2}$ (Table 2).

\section{Discussion}

The present study demonstrates, in a population of 563 patients followed for about 5 years after cardiac surgery, that post-operative LAVi is a long-term predictor of MACCEs and CV mortality, also confirming its prognostic value in predicting MACCEs even after adjustment for age, gender, diabetes, atrial fibrillation at discharge, E/A diastolic index and LVEF.

Left atrium reflects left ventricular filling pressure, a parameter conditioned by structural and functional heart impairement $[1,2]$. Consequentely, LA enlargement is an echocardiographic marker that mirrors left ventricular systolic and/or diastolic chronic dysfunction, representing a possible pathophysiological explanation of our results.

Previous studies in patients undergoing cardiac surgery only examined the relationship between LA volume and prevalence of either post-operative atrial fibrillation [13] or symptomatic improvement of HF
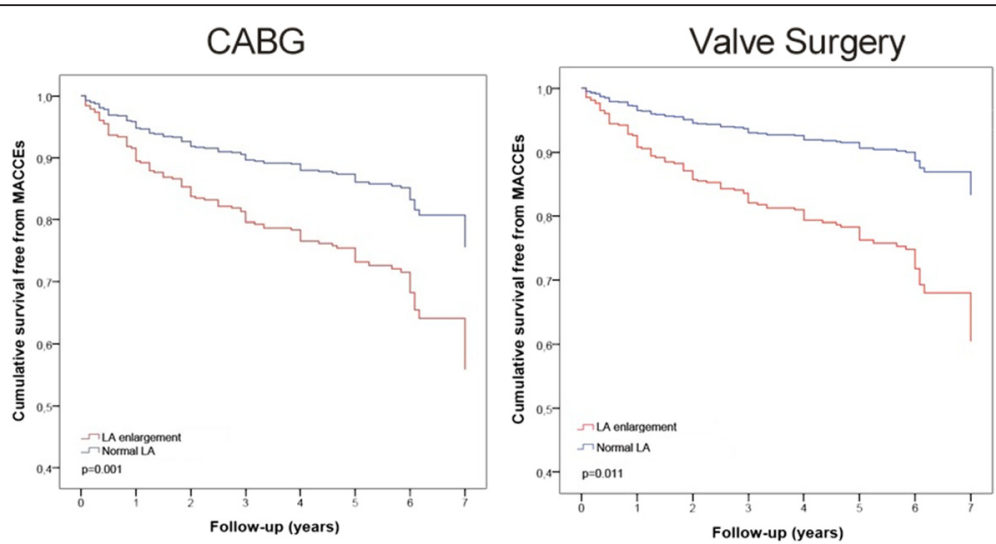

Fig. 1 Left: LA enlargement and survival free from MACCEs in CABG patients. Right: LA enlargement and survival free from MACCEs in valve surgery patients. MACCEs, major adverse cardiovascular and cerebrovascular events; LA, left atrium 


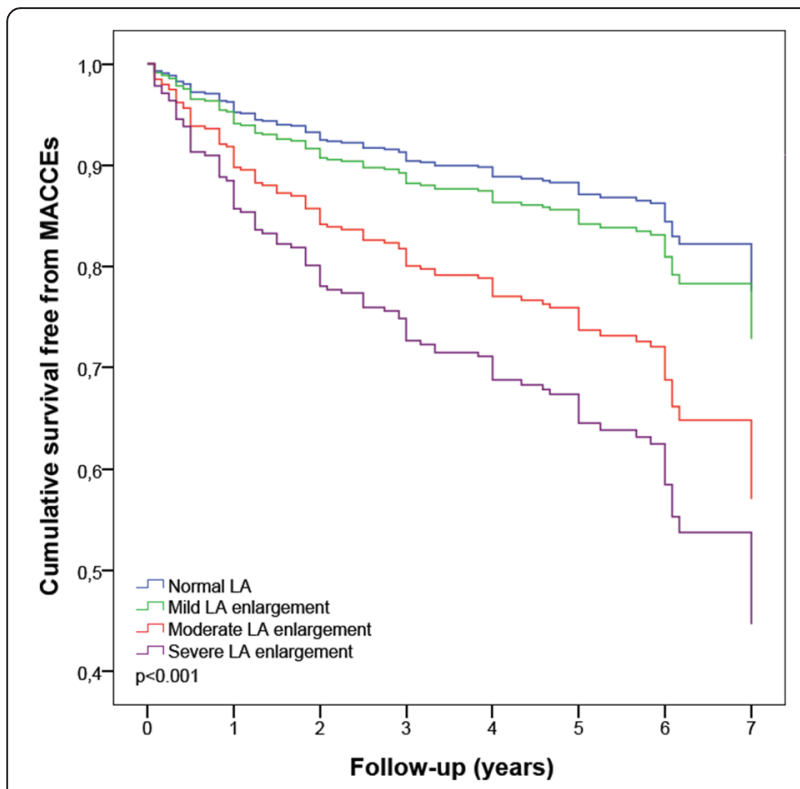

Fig. 2 MACCES and LA severity partition cut-offs after cardiac surgery. MACCES, major adverse cardiovascular and cerebrovascular events; LA, left atrium

[14]. Therefore, our results offer novel data highlighting a significant prognostic role of LAVi on MACCEs and CV mortality in such patients. In addition, our study represents a convincing validation of the prognostic significance of the new severity partition cutoffs, suggested by

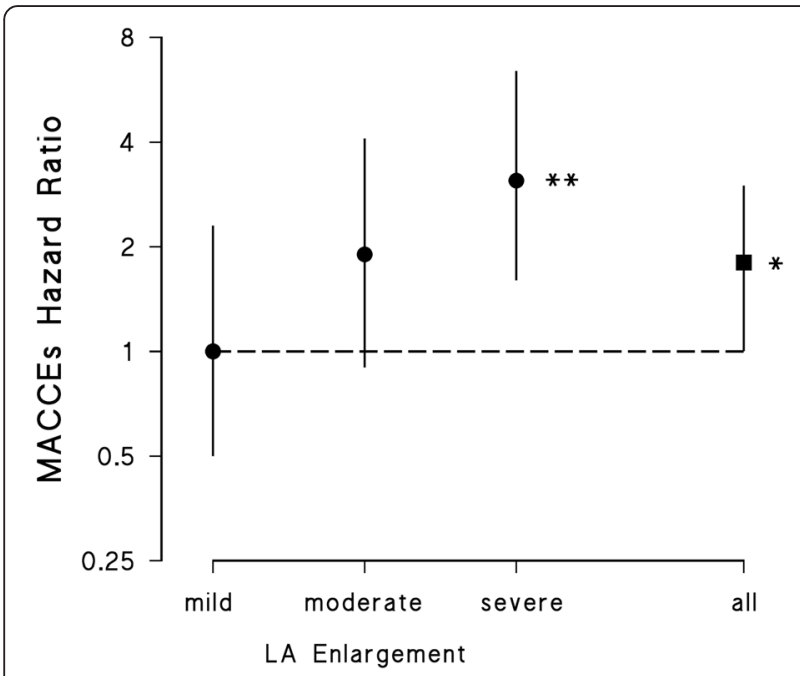

Fig. 3 Hazard Ratio with $95 \%$ Cl for MACCEs calculated after adjustment for age, LVEF, gender, diabetes, atrial fibrillation at discharge and E/A ratio, in subgroups of patients with mild, moderate and severe LA enlargement, and in all patients with LA enlargement; * and ** indicate significant differences from $\mathrm{HR}=1$ at $p<0.05$ and $p<0.01$ significance levels both EACVI and ASE [12], in patients undergoing cardiac surgery. In particular, we showed that moderate and severe LA enlargement is associated with increased rate of MACCEs, while mildly increased LAVi represents a grey zone which does not appear to robustly impact the prognosis.

LA size is a recognized prognostic predictor in primary CV prevention. This was demonstrated in a 8year follow-up of 5209 individuals included in the Framingham Heart Study, where an increased LA diameter was associated with higher risk of stroke and mortality [15] and other primary prevention studies suggested the independent role of LAVi in predicting $\mathrm{CV}$ death $[3,5,7]$, MACCEs $[5,7]$, ischemic stroke [6] and atrial fibrillation [4].

The prognostic significance of LAVi has been confirmed also in secondary CV prevention, especially in patients after acute myocardial infarction (MI). An increased LA volume, assessed within the first $48 \mathrm{~h}$ of admission, independently predicted 5 -year mortality in a population of 395 MI patients [8]. Similarly, LAVi $>32 \mathrm{ml} / \mathrm{m}^{2}$ did result an independent predictor of mortality during a mean follow-up of 15 months in 314 MI patients [16], being these results thereafter confirmed in a population of 610 subjects with highrisk MI followed for a mean of 20 months [9]. The relationship between LAVi and CV outcomes was also addressed in 935 outpatients with established coronary artery disease followed for about 4 years, concluding that LAVi was similar to LVEF in predicting mortality and HF hospitalization [17].

The prognostic implications of LA size were confirmed also in patients with valve disease, who did or did not undergo cardiac surgery. Neverthless, the majority of these studies has been mainly focused on post-operative atrial fibrillation $[13,18,19]$, LVEF recovery [20] and HF symptoms [14], while harder endpoints, such as cardiovascular mortality, had only been evaluated in 176 patients with symptomatic chronic mitral regurgitation undergoing valve replacement by using pre-operative LA diameter [21].

Given that in previous studies the predictive significance of LA size was invariably investigated measuring LA diameter or LAVi with either arbitrary or single cutoff value, our study could represent the first prognostic validation of the new three cutoffs of severity partition, recently recommended by both EACVI and ASE. Our results highlight the importance to quantify the LA size in terms of LAVi; they also suggest that LAVi should be measured not only in routine clinical practice but also in a rehabilitation setting for more accurate risk-stratification and clinical decision making in patients who underwent cardiac surgery. 

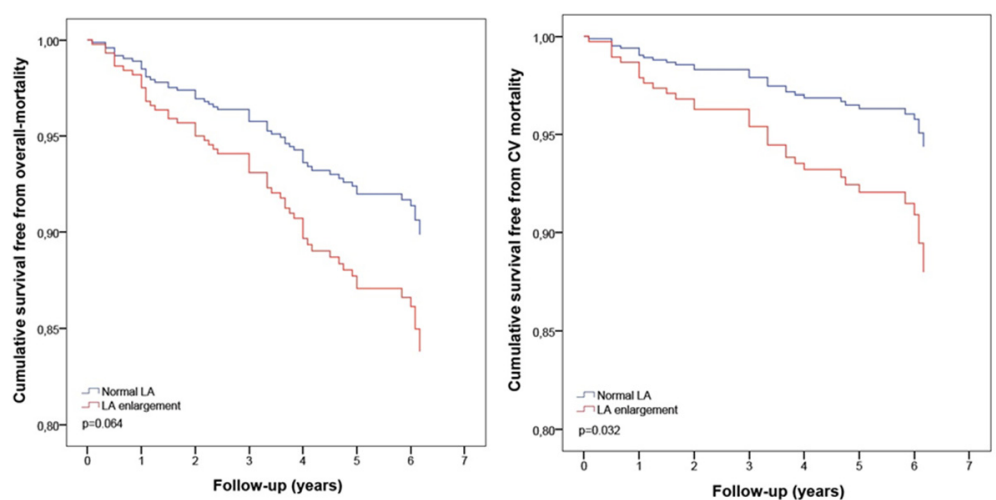

Fig. 4 Left: Overall mortality and LA enlargement. Right: CV mortality and LA enlargement. LA, left atrium; CV, cardiovascular

\section{Study limitations}

The entry criterion for the study was the availability of LAVi measurement and in our registry of 1703 patients, only 563 had such data available for analysis; this approach, driven by the absence of a protocol for LA measurement using LAVi, may have introduced a selection bias.

Although this intrinsic bias could not reflect the real prevalence of LA dimension in the whole population, the rate of LA enlargement, assessed in the entire cohort with LA diameter (cut-off $40 \mathrm{~mm}$ ), was similar to that evaluated in the subgroup of patients with available biplane left LA volume data ( $46 \%$ of LA enlargement using LAVi and $48 \%$ using LA dimeter).

Given the retrospective nature of the study, LAVi measurements were obtained by multiple observers working in the same clinical environment and using the same echo machine. This may have created an inter-observer bias related to the variability of the measurements; on the other hand our results would suggest that the prognostic significance of LAVi can be widely applied in the clinical setting.

\section{Conclusions}

Increased post-operative LAVi represents a predictor of adverse outcome after cardiac surgery and provides prognostic information, importantly incremental to several conventional risk factors. The recently recommended LAVi severity cutoffs appear adequate to effectively stratify outcome in patients undergoing rehabilitation after cardiac surgery. These results should be verified trough a prospective, large scale multicentre study design.

\section{Acknowledgement}

None.

\section{Funding}

None.

\section{Availability of data and material}

Yes.

\section{Authors' contributions}

The manuscript has been read and approved by all the authors, the requirements for authorship have been met and each author believes that the manuscript represents an original and honest work.

\section{Competing interests}

None.

\section{Consent for publication}

Yes.

Ethics approval and consent to participate

Yes.

\section{Author details}

${ }^{1}$ Fondazione Don Carlo Gnocchi, University of Parma, Fondazione Don Gnocchi, Piazzale dei servi n 3, 43121 Parma, Italy. ${ }^{2}$ Department of Cardiology, Parma University Hospital, Parma, Italy. ${ }^{3}$ Department of Clinical and Experimental Medicine, University of Parma, Parma, Italy. ${ }^{4}$ RCCS Fondazione Don C. Gnocchi, Milan, Italy.

Received: 30 June 2016 Accepted: 13 August 2016

Published online: 23 August 2016

References

1. Barbier P, Solomon SB, Schiller NB, Glantz SA. Left atrial relaxation and left ventricular systolic function determine left atrial reservoir function. Circulation. 1999;100:427-36.

2. Douglas PS. The left atrium: a biomarker of chronic diastolic dysfunction and cardiovascular disease risk. J Am Coll Cardiol. 2003;42:1206-7.

3. Leung DY, Chi C, Allman C, Boyd A, Ng AC, Kadappu KK, et al. Prognostic implications of left atrial volume index in patients in sinus rhythm. Am J Cardiol. 2010:105:1635-9.

4. Abhayaratna WP, Fatema K, Barnes ME, Seward JB, Gersh BJ, Bailey KR, et al. Left atrial reservoir function as a potent marker for first atrial fibrillation or flutter in persons > 65 years of age. Am J Cardiol. 2008;101:1626-9.

5. Tsang TS, Abhayaratna WP, Barnes ME, Miyasaka Y, Gersh BJ, Bailey KR, et al. Prediction of cardiovascular outcomes with left atrial size: is volume superior to area or diameter? J Am Coll Cardiol. 2006;47:1018-23.

6. Barnes ME, Miyasaka Y, Seward JB, Gersh BJ, Rosales AG, Bailey KR, et al. Left atrial volume in the prediction of first ischemic stroke in an elderly cohort without atrial fibrillation. Mayo Clin Proc 2004:79:1008-14.

7. Bombelli M, Facchetti R, Cuspidi C, Villa P, Dozio D, Brambilla G, et al. Prognostic significance of left atrial enlargement in a general population. Results of the PAMELA study. Hypertension. 2014;64:1205-11. 
8. Beinart R, Boyko V, Schwammenthal E, Kuperstein R, Sagie A, Hod H, et al. Long-term prognostic significance of left atrial volume in acute myocardial infarction. J Am Coll Cardiol. 2004;44:327-34.

9. Meris A, Amigoni M, Uno H, Thune JJ, Verma A, Køber L, et al. Left atrial remodeling in patients with myocardial infarction complicated by heart failure, left ventricular dysfunction, or both: the VALIANT Echo study. Eur Heart J. 2009;30:56-65.

10. Tani T, Yagi T, Kitai T, Kim K, Nakamura H, Konda T, et al. Left atrial volume predicts adverse cardiac and cerebrovascular events in patients with hypertrophic cardiomyopathy. Cardiovasc Ultrasound. 2011;9:34. doi:10. 1186/1476-7120-9-34

11. Eirin S, Akira Y, Kunihico S, Yoshihiro I, Kenji S, Kayoko T, et al. Prognostic value of left atrial volume index in patients with first acute myocardial infarction. Eur J Echocardiogr. 2011;12:440-4.

12. Lang RM, Badano LP, Mor-Avi V, Afilalo J, Armstrong A, Ernande L, et al. Recommendations for cardiac chamber quantification by echocardiography in adults: an update from the american society of echocardiography and the European Association of cardiovascular imaging. Eur Heart I Cardiovasc Imaging. 2015;16(3):233-70. doi:10.1093/ehjci/jev014.

13. Osranek M, Fatema K, Qaddoura F, Al-Saileek A, Barnes ME, Bailey KR, et al. Left atrial volume predicts the risk of atrial fibrillation after cardiac surgery: a prospective study. J Am Coll Cardiol. 2006;48:779-86.

14. Rossi A, Tomaino M, Golia G, Santini F, Pentiricci S, Marino P, et al. Usefulness of left atrial size in predicting postoperative symptomatic improvement in patients with aortic stenosis. Am J Cardiol. 2000;86:567-70.

15. Benjamin EJ, D'Agostino RB, Belanger AJ, Wolf PA, Levy D. Left atrial size and the risk of stroke and death. The Framingham Heart study. Circulation. 1995;92:835-41.

16. Møller JE, Hillis GS, Oh JK, Seward JB, Reeder GS, Wright RS, et al. Left atria volume: a powerful predictor of survival after acute myocardial infarction. Circulation. 2003;107:2207-12.

17. Ristow B, Ali S, Whooley MA, Schiller NB. Usefulness of left atrial volume index to predict heart failure hospitalization and mortality in ambulatory patients with coronary heart disease and comparison to left ventricular ejection fraction (from the Heart and Soul Study). Am J Cardiol. 2008;102:70-6.

18. Naito Y, Yamazaki K. Preoperative left atrial volume index predicts postoperative atrial fibrillation in patients with severe aortic valve stenosis. J Anesth. 2013:27:699-04.

19. Candan O, Ozdemir N, Aung SM, Dogan C, Karabay CY, Gecmen C, et al. Left atrial longitudinal strain parameters predict postoperative persistent atrial fibrillation following mitral valve surgery: a speckle tracking echocardiography study. Echocardiography. 2013;30:1061-8.

20. Cho IJ, Chang HJ, Hong GR, Heo R, Sung JM, Lee SE, et al. Left atrial volume index as a predictor for persistent left ventricular dysfunction after aortic valve surgery in patients with chronic aortic regurgitation: the role of early postoperative echocardiography. Echocardiography. 2015;32:896-03.

21. Reed D, Abbott RD, Smucker ML, Kaul S. Prediction of outcome after mitral valve replacement in patients with symptomatic chronic mitral regurgitation. The importance of left atrial size. Circulation. 1991;84:23-34.

\section{Submit your next manuscript to BioMed Central and we will help you at every step:}

- We accept pre-submission inquiries

- Our selector tool helps you to find the most relevant journal

- We provide round the clock customer support

- Convenient online submission

- Thorough peer review

- Inclusion in PubMed and all major indexing services

- Maximum visibility for your research

Submit your manuscript at www.biomedcentral.com/submit

) Biomed Central 